\title{
A simple dynamic model for mitochondrial metabolism
}

\author{
G. Sváb* G. Szederkényi ${ }^{* *}$ G. Horvath ${ }^{*}$ L. Tretter * \\ * Department of Medical Biochemistry, Semmelweis University, \\ Budapest, Hungary, (e-mail: gergely@svab.hu) \\ ** Faculty of Information Technology and Bionics, Pázmány Péter \\ Catholic University, Budapest, Hungary (e-mail: \\ szederkenyi@itk.ppke.hu)
}

\begin{abstract}
A simple dynamical model is proposed in this paper to describe the main timevarying quantities in mitochondrial metabolism. The model is given in the form of kinetic ordinary differential equations containing 11 state equations. The parameters of the model were determined from the literature or from the authors' own laboratory measurements. The obtained simulation result is in good agreement with actual observation for simulating an ischemic process.
\end{abstract}

Keywords: biomedical systems, dynamical modeling, simulation, differential equations

\section{INTRODUCTION}

In the Neurobiochemistry working group (Institute of Medical Biochemistry, Semmelweis University), we have taken enzyme kinetic measurements of several mitochondrial enzymes, and we have determined and analyzed interactions between enzymes and substrates. Therefore, a suitable quantitative (mathematical) model is constructed which describes the temporal changes in the quantities of citric acid cycle's (CAC) molecules. The modeling goal in this research phase is to describe qualitatively the increased/decreased operation of the catalyzing enzymes as well as the modified operation of the intermediate molecules.

In previous studies similar models were constructed, it belongs to our goals is to promote further development compared to the known models and to expand them in other aspects. In $\mathrm{Wu}$ et al. (2007) the model can predict the individual factors effects (NADH, ATP, metabolic fluxes) in the regulation of $\mathrm{CAC}$ function and projects the effect of $\mathrm{pH}$ and membrane potential changes on ATP synthesis. In Korla and Mitra (2014) examination of the process of the CAC, electron transport chain and ATP synthesis is described. Reactions of the CAC are described with eight differential equations with Michaelis-Menten reaction kinetic. Korla et al. (2015) extends the earlier model with two transporter systems.

\section{BIOLOGICAL BACKGROUND}

\subsection{Process description}

Mitochondria are cell organelles with prokaryotic origin that established endosymbiosis with ancient eukaryotic

\footnotetext{
* The project has been partially supported by the European Union, co-financed by the European Social Fund through the grants EFOP-3.6.3-VEKOP-16-2017-00002 and EFOP-3.6.3-VEKOP-162017-00009
}

cells during evolution. CAC is a complex enzyme system in mitochondria, and a key step of metabolism. Mitochondria produce energy during the catabolic degradation of carbohydrates, fats, proteins and nucleic acids. Additionally, molecules of the CAC take part as precursors in the construction of the anabolic procedures (Nelson and Cox (2012)).

\subsection{The modeled reactions}

In this work we modeled the reactions of $\mathrm{CAC}$, and 3 mitochondrial transporters. The following list contains the reactions and their catalyzing enzymes/transporters involved in our model (Number of reaction, name of enzyme, Enzyme Commission (EC) number of enzyme).

R1: Citrate synthase (CS, 4.1.3.7):

Acetyl-CoA + oxaloacetate $\rightarrow$ citrate

R2: Aconitase (ACON, 4.2.1.3):

Citrate $\rightleftharpoons$ isocitrate

R3: Isocitrate dehydrogenase (IDH, 1.1.1.41):

Isocitrate $+\mathrm{NAD}^{+} \rightarrow$ oxoglutarate $+\mathrm{CO}_{2}+\mathrm{NADH}$

R4: Oxoglutarate dehydrogenase (OGDH, 1.2.4.2):

Oxoglutarate $+\mathrm{CoA}-\mathrm{SH}+\mathrm{NAD}^{+} \rightarrow$ succinyl-CoA $+\mathrm{CO}_{2}+$ $N A D H$

R5: Succinyl-Coenzyme A ligase (SUCLA, 6.2.1.4):

Succinyl-CoA + GDP $+P \rightarrow$ succinate $+C o A-S H+G T P$

R6: Succinate dehydrogenase (SDH, 1.3.5.1):

Succinate $+F A D \rightarrow$ fumarate $+F_{A D H}$

R7: Fumarase (FH, 4.2.1.2):

Fumarate $+\mathrm{H}_{2} \mathrm{O} \rightleftharpoons$ malate

R8: Malate dehydrogenase (MDH, 1.1.1.37):

Malate $+\mathrm{NAD}^{+} \rightleftharpoons$ oxaloacetate $+\mathrm{NADH}$

R9: Citrate transporter (CTP):

Citrate $+H^{+} \rightleftarrows$ malate

R10: Dicarboxylate carrier (DIC):

$\mathrm{HPO}_{4}^{2-} \rightleftarrows$ malate

R11: Oxoglutarate carrier (OGC):

Oxoglutarate $\rightleftarrows$ malate 


\section{KINETIC MODEL OF CITRIC-ACID CYCLE}

\subsection{Modeling goals}

The modeling goals are the following:

(1) To determine the concentrations of substrates which participate in these reactions between physiological conditions.

(2) To predict the new equilibrium status after we modified initial conditions.

(3) To model the changed dynamic of the system in case of specific diseases via using pathological parameters of enzymes and/or pathological initial conditions.

\subsection{Parameters}

Table 1 contains the kinetic parameters. Source of parameters is the literature and some of them were measured in Institute of Medical Biochemistry, Semmelweis University, namely the values corresponding to $\mathrm{OGDH}, \mathrm{SDH}$ and $\mathrm{MDH}$.

\begin{tabular}{|c|c|c|c|}
\hline E or T & substrate & $\mathrm{V}_{\max }(\mu \mathrm{mol} / \mathrm{min})$ & $\mathrm{K}_{M}(\mu \mathrm{M})$ \\
\hline \hline CS & oxaloacetate & 1.88 & 4 \\
\hline ACON & citrate & 3.4 & 470 \\
& isocitrate & & 120 \\
\hline IDH & isocitrate & 0.1 & 140 \\
\hline OGDH & oxoglutarate & 0.0086 & 71 \\
\hline SUCLA & succinil-CoA & 0.39 & 40 \\
\hline SDH & succinate & 0.299 & 251 \\
\hline FH & fumarate & 0.721 & 13 \\
& malate & & 140 \\
\hline MDH & malate & 1.616 & 580 \\
& oxaloacetate & & 52 \\
\hline CTP $(\mathrm{T})$ & citrate & 10.5 & $32 / 27$ \\
& malate & 11.5 & $250 / 60$ \\
\hline DIC $(\mathrm{T})$ & malate & 6 & $490 / 920$ \\
& HPO & & $1410 / 930$ \\
\hline OGC $(\mathrm{T})$ & oxoglutarate & 6.5 & $310 / 170$ \\
& malate & 10 & $1360 / 710$ \\
\hline
\end{tabular}

Table 1. Parameters and values of enzymes and transporters

\subsection{The applied kinetics}

Differential equations were described by parameters of enzymes and transporters using the Michaelis-Menten kinetic model. The general form of the reaction rates is

$$
V([S])=\frac{V_{\max } \cdot[S]}{K_{M}+[S]}
$$

Rates of reversible reactions and transports are described with two opposite reactions. We assume constant cell concentrations, because the volume of cell is much larger than that of the mitochondrium.

\section{COMPUTATIONAL RESULTS FOR SIMULATING REGENERATION AFTER ISCHEMY}

The simulations were implemented in MATLAB. We examine a pathological metabolic status after acute ischemy. In this state there is a significant change in substrate

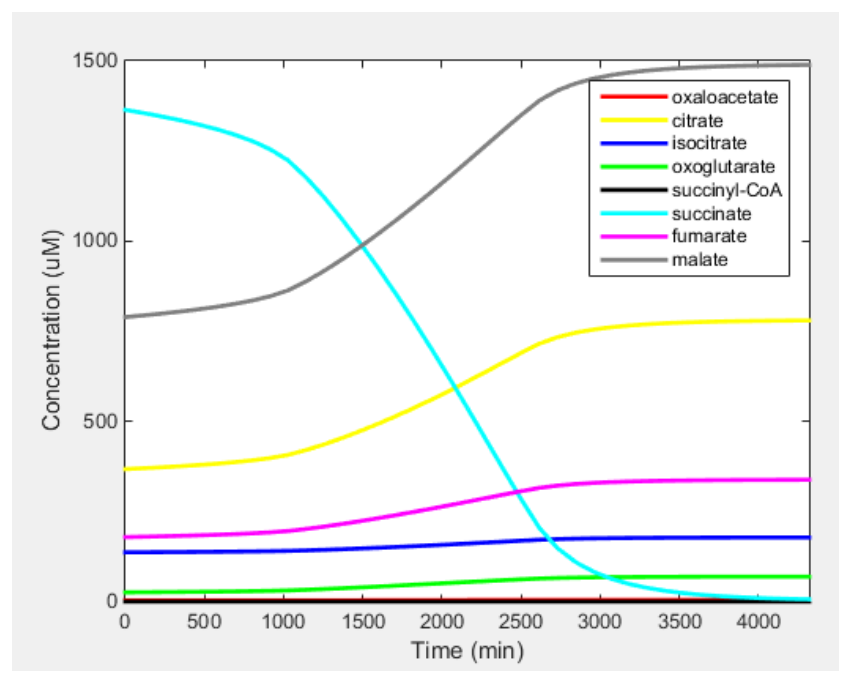

Fig. 1. Changes of concentrations after acute ischemia

concentrations. Based on literature data, the altered initial conditions were the following: citrate decreases to $370 \mu \mathrm{M}$, oxoglutarate to $26 \mu \mathrm{M}$, fumarate to $180 \mu \mathrm{M}$, malate to 795 $\mu \mathrm{M}$, and succinate to $1355 \mu \mathrm{M}$.

The simulation shows that after ischemic status concentrations of substrates are going to physiological concentrations, as the system tries to recover physiological status (Figure 1). Moreover, we can observe that approximately three days are needed to restore normal concentrations of mitochondrial intermediates. These results show us that this model gives good results qualitatively, but further improvements are needed by parameter calibration and by the extension with other transporters.

\section{CONCLUSION}

A simple dynamic model for describing the dynamics of key quantities in mitochondrial metabolism was proposed in this paper. The model is written in the form of 12 nonlinear ODEs using Michaelis-Menten kinetics. The model was used successfully to simulate recovery from ischemy.

\section{REFERENCES}

Korla, K. and Mitra, C. (2014). Modelling the krebs cycle and oxidative phosphorylation. Journal of Biomolecular Structure and Dynamics, 32, 242-256.

Korla, K., Vadlakonda, L., and Mitra, C. (2015). Kinetic simulation of malate-aspartate and citrate-pyruvate shuttles in association with krebs cycle. Journal of Biomolecular Structure and Dynamics, 33(11), 2390-403.

Nelson, D.L. and Cox, M.M. (2012). Lehninger Principles of Biochemistry. WH Freeman, 6 edition.

Wu, F., Yang, F., Vinnakota, K., and Beard, D. (2007). Computer modeling of mitochondrial tricarboxylic acid cycle, oxidative phosphorylation, metabolite transport, and electrophysiology. The Journal of Biological Chemistry, 282(34), 24525-37. 